\title{
Optimal Control Problem for Uncertain Linear Systems with Multiple Input Delays
}

\author{
Yi Jiang ${ }^{1}$, Hongyan Yan ${ }^{2}$ and Yuanguo Zhu ${ }^{1 *}$
}

*Correspondence:
ygzhu@njust.edu.cn
'School of Science, Nanjing
University of Science and
Technology, 210094 Nanjing, China
Full list of author information is
available at the end of the article

available at the end of the article

\begin{abstract}
Based on the concept of uncertain process, uncertain linear systems with multiple input delays are considered in this paper. An optimal problem for such systems is introduced and solved by using the equation of optimality and maximum principle. Finally, an example is given to show how to solve an uncertain optimal control model with time-delay.
\end{abstract}

Keywords: Optimal control, Uncertain system, Input delay

\section{Introduction}

The delay system was first proposed in the eighteenth century. Until the twentieth century, with the maximum principle and dynamic programming theory, delay system attracted significant interest from many researchers. In recent decades, the study of timedelay systems had a great development not only in theory but also in applications. Optimal control problems of linear time-delay systems were discussed in Basin [1] and Eller et al. [2]. Stochastic control problems with delay were studied by Bauer and Rieder [3] and Larssen and Risebro [4]. A set of infinite-dimensional differential equations could model the dynamic systems which have multiple input delays. Hamiltonian function can be used to solve the control problems with delay [5]. Moreover, the phenomenon of systems with multiple input delays are ubiquitous. It widely exists in communications. With the rapid development of information and technology, multi-input time-delay systems become a research hot spot.

In the real world, we will encounter a lot of indeterminacy; these phenomena cannot be explained by random events, such as high speed, about $50 \mathrm{~kg}$. This fact prompted researchers to create a new mathematical tool. Liu [6] found an uncertainty theory through introducing an uncertain measure based on normality, self-duality, countable subadditivity, and product measure axioms. Based on an uncertain variable, uncertain process, and canonical process, Liu [7] presented uncertain differential equation. It aims to describe the evolution of dynamic uncertain systems and has widely applied in finance so far. Liu [8] assumed the stock price follows a time-homogenous uncertain differential equation and proposed the first uncertain stock model. Ji and Zhou [9] proved the existence and uniqueness of a multi-dimensional uncertain differential equation. Based on the uncertain differential equation, in 2010, Zhu [10] presented an equation of optimality for an uncertain optimal control problem by using dynamic programming. This equation

(c) 2016 The Author(s). Open Access This article is distributed under the terms of the Creative Commons Attribution 4.0 International License (http://creativecommons.org/licenses/by/4.0/), which permits unrestricted use, distribution, and reproduction in any medium, provided you give appropriate credit to the original author(s) and the source, provide a link to the Creative Commons license, and indicate if changes were made. 
plays a significant role in solving uncertain optimal control problems. It has been applied to uncertain bang-bang control problems by $\mathrm{Xu}$ and $\mathrm{Zhu}$ [11] and uncertain time-delay systems control problem by Chen and Zhu [12].

In this paper, we will introduce an uncertain linear system with multiple input delays and propose an optimal control problem subject to this system. The organization of the paper is as follows. In the "Preliminary" section, some basic concepts are reviewed. In the "Problem Statement" section, an uncertain linear system with multiple input delays is formulated, and the optimal control is derived in the problem by using the equation of optimality. In the "Example" section, we will give a numerical example of uncertain linear systems with multiple input delays to illustrate the result obtained in the previous section.

\section{Preliminary}

Some concepts about uncertain measure, uncertain variable, and uncertain process can be found in Liu [6]. In convenience, we give some useful concepts. Let $\Gamma$ be a nonempty set and $L$ be a $\sigma$-algebra over $\Gamma$. The set function $M$ defined on the $\sigma$-algebra $L$ is called an uncertain measure if it satisfies the three axioms: $M\{\Gamma\}=1 ; M\{\Lambda\}+M\left\{\Lambda^{c}\right\}=1$ for any event $\Lambda \in L$; and $M\left\{\bigcup_{i=1}^{\infty} \Lambda_{i}\right\} \leq \sum_{i=1}^{\infty} M\left\{\Lambda_{i}\right\}$ for every countable sequence of events $\left\{\Lambda_{i}\right\} \subset L$. Then, the triplet $(\Gamma, L, M)$ is called an uncertainty space. An uncertain variable is a measurable function from an uncertainty space $(\Gamma, L, M)$ to the set $R$ of real numbers, and an uncertain vector is a measurable function from an uncertainty space to $R^{n}$. The uncertainty distribution $\Phi: R \rightarrow[0,1]$ of an uncertain variable $\xi$ is defined by $\Phi(x)=M\{\xi \leq x\}$ for any real number $x$. An uncertain process is a measurable function from $V \times(\Gamma, L, M)$ to the set of real numbers where $V$ is an index set.

Definition 1 (Liu [8]) The uncertain variables $\xi_{1}, \xi_{2}, \cdots \xi_{m}$ are said to be independent if $M\left\{\bigcap_{i=1}^{m}\left(\xi_{i} \in B_{i}\right)\right\}=\min _{1 \leq i \leq m} M\left\{\xi_{i} \in B_{i}\right\}$ for any Borel sets $B_{1}, B_{2}, \cdots B_{m}$ of real numbers.

Definition 2 (Liu [6]) The expected value of an uncertain variable $\xi$ is defined by

$$
E[\xi]=\int_{0}^{+\infty} M\{\xi \geq r\} d r-\int_{-\infty}^{0} M\{\xi \leq r\} d r,
$$

provided that at least one of the two integrals is finite.

For any numbers $a$ and $b, E[a \xi+b \eta]=a E[\xi]+b E[\eta]$ if $\xi$ and $\eta$ are independent uncertain variables.

Definition 3 (Liu [8]) An uncertain process $C_{t}$ is said to be a canonical process if (i) $C_{0}=0$ and almost all sample paths are Lipschitz continuous; (ii) $C_{t}$ has stationary and independent increments; (iii) every increment $C_{s+t}-C_{s}$ is a normal uncertain variable with expected value 0 and variance $t^{2}$, denoted by $C_{s+t}-C_{s} \sim N(0, t)$, whose distribution is

$$
\Phi(x)=\left(1+\exp \left(\frac{-\pi x}{\sqrt{3} t}\right)\right)^{-1}, x \in R
$$

Definition 4 (Liu [8]) Let $X_{t}$ be an uncertain process and $C_{t}$ be a canonical process. For any partition of closed interval $[a, b]$ with $a=t_{1}<t_{2}<\cdots<t_{k+1}=b$, the mesh 
is written as $\Delta=\max _{1 \leq i \leq k}\left|t_{i+1}-t_{i}\right|$. Then, uncertain integral of $X_{t}$ with respect to $C_{t}$ is

$$
\int_{a}^{b} X_{t} d C_{t}=\lim _{\triangle \rightarrow 0} \sum_{i=1}^{k} X_{t_{i}} \cdot\left(C_{t_{i+1}}-C_{t_{i}}\right)
$$

provided that the limit exists almost surely and is finite.

Definition 5 (Liu [8]) Suppose $C_{t}$ is a canonical process, and $f$ and $g$ are two functions. Then,

$$
d X_{t}=f\left(t, X_{t}\right) d t+g\left(t, X_{t}\right) d C_{t}
$$

is called an uncertain differential equation. A solution is an uncertain process $X_{t}$ that satisfies (1) identically in $t$.

Remark The uncertain differential Eq. (1) is equivalent to the uncertain integral equation

$$
X_{s}=X_{0}+\int_{0}^{s} f\left(t, X_{t}\right) d t+\int_{0}^{s} g\left(t, X_{t}\right) d C_{t} .
$$

\section{Problem Statement}

Consider an uncertain linear system with multiple time-delays in the control input

$$
d X_{s}=\left(a_{0}(s)+a_{1}(s) X_{s}+\sum_{i=1}^{p} B_{i}(s) u\left(s-h_{i}\right)\right) d s+b(s) d C_{s},
$$

with the initial condition $X\left(t_{0}\right)=X_{0}$, where $t_{0}$ is the initial time. Here, $X_{s}$ is the state vector of $n$ dimension, $u_{s}$ is the control vector of $m$ dimension; $h_{i}>0(i=$ $1, \cdots, p)$ are positive time-delays; $h=\max \left\{h_{1}, \cdots, h_{p}\right\}$ is the maximum delay shift; and $C_{s}=\left(C_{s_{1}}, C_{s_{2}}, \cdots, C_{s_{p}}\right)$, where $C_{s_{1}}, C_{s_{2}}, \cdots, C_{s_{p}}$ are independent canonical process. And $a_{0}(s), a_{1}(s), b(s)$, and $B_{i}(s)(i=1,2, \cdots, p)$ are piecewise continuous matrix functions of appropriate dimensions.

The quadratic cost function to be maximized is defined as follows:

$$
J\left(t, X_{t}\right)=\sup _{u \in U} E\left(\frac{1}{2} \int_{t}^{T}\left(u_{s}^{\tau} R(s) u_{s}+X_{s}^{\tau} L(s) X_{s}\right) d s+X_{T}^{\tau} \Psi_{T} X_{T}\right),
$$

where $R(s)$ is positive definite, $\Psi_{T}, L(s)$ are nonnegative definite symmetric matrices, and $T>0$ is a time moment.

The optimal control problem is to find the control $u^{*}(t)\left(t \in\left[t_{0}, T\right]\right)$ that maximizes the criterion $J(t, x)$ along with the trajectory $x^{*}(t)\left(t \in\left[t_{0}, T\right]\right)$, generated upon substituting $u^{*}(t)$ into the state Eq. (2).

Theorem 1 Let $C_{t}$ be a canonical process, $u_{1 t}$ be an $n \times n$ integrable uncertain process, and $u_{2 t}$ and $v_{2 t}$ be two $n$-dimensional integrable uncertain processes. Then, the $n$-dimensional linear uncertain differential equation

$$
d X_{t}=\left(u_{1 t} X_{t}+u_{2 t}\right) d t+v_{2 t} d C_{t}
$$


has a solution

$$
X_{t}=U_{t}\left(X_{0}+\int_{0}^{t} U_{s}^{-1} u_{2 s} d s+\int_{0}^{t} U_{s}^{-1} v_{2 s} d C_{s}\right),
$$

where

$$
U_{t}=\exp \left(\int_{0}^{t} u_{1 s} d s\right)
$$

Proof At first, we define two uncertain processes $U_{t}$ and $V_{t}$ via uncertain differential equations,

$$
d U_{t}=u_{1 t} U_{t} d t, d V_{t}=U_{t}^{-1} u_{2 t} d t+U_{t}^{-1} v_{2 t} d C_{t} .
$$

It follows from the integration by parts that

$$
d\left(U_{t} V_{t}\right)=U_{t} d V_{t}+d U_{t} \cdot V_{t}=\left(u_{1 t} U_{t} V_{t}+u_{2 t}\right) d t+\left(v_{2 t}\right) d C_{t} .
$$

That is, the uncertain process $X_{t}=U_{t} V_{t}$ is a solution of the uncertain differential Eq. (4). Write $U_{t}$ as

$$
U_{t}=\sum_{n=0}^{\infty} \frac{1}{n !}\left(\int_{0}^{t} u_{1 s} d s\right)^{n} \cdot U_{0}
$$

Taking differentiation operations on both sides, we have

$$
d U_{t}=\sum_{n=1}^{\infty} \frac{u_{1 t}}{(n-1) !}\left(\int_{0}^{t} u_{1 s} d s\right)^{n-1} d t \cdot U_{0}=u_{1 t} \sum_{n=0}^{\infty} \frac{1}{n !}\left(\int_{0}^{t} u_{1 s} d s\right)^{n} \cdot U_{0} d t=u_{1 t} U_{t} d t .
$$

Thus, the solution of uncertain differential equation $d U_{t}=u_{1 t} U_{t} d t$ is

$$
U_{t}=\sum_{n=0}^{\infty} \frac{1}{n !}\left(\int_{0}^{t} u_{1 s} d s\right)^{n} \cdot U_{0}=\exp \left(\int_{0}^{t} u_{1 s} d s\right) \cdot U_{0} .
$$

In addition, we have

$$
V_{t}=V_{0}+\int_{0}^{t} U_{s}^{-1} u_{2 s} d s+\int_{0}^{t} U_{s}^{-1} v_{2 s} d C_{s} .
$$

Taking $U_{0}=I$ and $V_{0}=X_{0}$, we get the solution (5). The theorem is proved.

Theorem 2 The solution to the optimal control problem for the uncertain linear system with input delay (2) and the quadratic criterion (3) are given as follows. The optimal control law for $t \geq t_{0}$ is given by

$$
u^{*}(t)=-R^{-1}(t) \sum_{i=1}^{p} B_{i}(t) M_{i}(t)(P(t) x+Q(t))
$$

where $P(t)$ satisfies

$$
\left\{\begin{array}{l}
\dot{P}(t)=-\frac{1}{2} \sum_{i=1}^{p} M_{i}^{\tau}(t) B_{i}^{\tau}(t) P^{\tau}(t) R^{-1}(t) P(t) \sum_{i=1}^{p} B_{i}(t) M_{i}(t)+L(t)+a_{1}(t) P(t) \\
P(T)=2 \Psi
\end{array}\right.
$$

and $Q(t)$ is a solution of the following differential equation

$$
\left\{\begin{array}{l}
\dot{Q}(t)=-\sum_{i=1}^{p} M_{i}^{\tau}(t) B_{i}^{\tau}(t) P(t) R^{-1}(t) Q(t) \sum_{i=1}^{p} B_{i}(t) M_{i}(t)+a_{0}(t) P(t)+a_{1}(t) Q(t) \\
Q(T)=0,
\end{array}\right.
$$


where $M_{i}(t)=\exp \left(-\int_{t-h_{i}}^{t} a_{1}(s) d s\right)$. The optimal value for $t \geq t_{0}$ is given by

$$
J(t, x)=\frac{1}{2} x^{\tau} P(t) x+Q(t) x+K(t)
$$

where

$$
K(t)=\int_{t}^{T}\left\{-\frac{1}{2} \sum_{i=1}^{p} M_{i}^{\tau}(s) B_{i}^{\tau}(s) Q(s)^{\tau} R^{-1}(s) Q(s) \sum_{i=1}^{p} B_{i}(s) M_{i}(s)+a_{0}(t) Q(s)\right\} d s .
$$

Proof Using the equation of optimality in Zhu [10] for the optimal control problems (2) and (3), we get

$$
-J_{t}\left(t, X_{t}\right)=\sup _{u \in U}\left\{\frac{1}{2}\left(u_{t}^{\tau} R(t) u_{t}+X_{t}^{\tau} L(t) X_{t}\right)+J_{x}^{\tau} a_{0}(t)+J_{x}^{\tau} a_{1}(t) X_{t}+J_{x}^{\tau} \sum_{i=1}^{p} B_{i}(t) u_{t-h_{i}}\right\} .
$$

Let

$$
g\left(u_{t}\right)=\frac{1}{2}\left(u_{t}^{\tau} R(t) u_{t}+X_{t}^{\tau} L(t) X_{t}\right)+J_{x}^{\tau} a_{0}(t)+J_{x}^{\tau} a_{1}(t) X_{t}+J_{x}^{\tau} \sum_{i=1}^{p} B_{i}(t) u_{t-h_{i}} .
$$

Setting $\frac{\partial g\left(u_{t}\right)}{\partial u_{t}}=0$ yields

$$
R(t) u_{t}+\sum_{i=1}^{p} M_{i}^{\tau}(t) B_{i}^{\tau}(t) J_{x}=0
$$

where $M_{i}(t)=\frac{\partial u_{t-h_{i}}}{\partial u_{t}}$. Hence,

$$
u_{t}^{*}=-R^{-1}(t) \sum_{i=1}^{p} M_{i}^{\tau}(t) B_{i}^{\tau}(t) J_{x} .
$$

By Eq. (9), we have

$$
-J_{t}=\frac{1}{2}\left(u_{t}^{* \tau} R(t) u_{t}^{*}+X_{t}^{\tau} L(t) X_{t}\right)+J_{x}^{\tau}\left(a_{0}(t)+a_{1}(t) X_{t}+\sum_{i=1}^{p} B_{i}(t) u_{t-h_{i}}^{*}\right) .
$$

Since $J\left(T, X_{T}\right)=X_{T}^{\tau} \Psi X_{T}$, we guess

$$
J(t, x)=\frac{1}{2} x^{\tau} P(t) x+Q(t) x+K(t) .
$$

Then,

$$
J_{t}=\frac{1}{2} x^{\tau} \dot{P}(t) x+\dot{Q}(t) x+\dot{K}(t),
$$

and

$$
J_{x}=P(t) x+Q(t) .
$$


Substituting Eqs. (10) and (13) into Eq. (11) yields

$$
\begin{aligned}
& -J_{t}\left(t, X_{t}\right)=x^{\tau}\left\{-\frac{1}{2} \sum_{i=1}^{p} M_{i}^{\tau}(t) B_{i}^{\tau}(t) P^{\tau}(t) R^{-1}(t) P(t) \sum_{i=1}^{p} B_{i}(t) M_{i}(t)+L(t)+a_{1}(t) P(t)\right\} x \\
& +\left\{-\sum_{i=1}^{p} M_{i}^{\tau}(t) B_{i}^{\tau}(t) P(t) R^{-1}(t) Q(t) \sum_{i=1}^{p} B_{i}(t) M_{i}(t)+a_{0}(t) P(t)+a_{1}(t) Q(t)\right\} x \\
& -\frac{1}{2} \sum_{i=1}^{p} M_{i}^{\tau}(t) B_{i}^{\tau}(t) Q(t)^{\tau} R^{-1}(t) Q(t) \sum_{i=1}^{p} B_{i}(t) M_{i}(t)+a_{0}(t) Q(t) .
\end{aligned}
$$

By Eqs. (12) and (14), we get

$$
\left\{\begin{array}{l}
\dot{P}(t)=-\frac{1}{2} \sum_{i=1}^{p} M_{i}^{\tau}(t) B_{i}^{\tau}(t) P^{\tau}(t) R^{-1}(t) P(t) \sum_{i=1}^{p} B_{i}(t) M_{i}(t)+L(t)+a_{1}(t) P(t) \\
\dot{Q}(t)=-\sum_{i=1}^{p} M_{i}^{\tau}(t) B_{i}^{\tau}(t) P(t) R^{-1}(t) Q(t) \sum_{i=1}^{p} B_{i}(t) M_{i}(t)+a_{0}(t) P(t)+a_{1}(t) Q(t),
\end{array}\right.
$$

and

$$
\dot{K}(t)=-\frac{1}{2} \sum_{i=1}^{p} M_{i}^{\tau}(t) B_{i}^{\tau}(t) Q(t)^{\tau} R^{-1}(t) Q(t) \sum_{i=1}^{p} B_{i}(t) M_{i}(t)+a_{0}(t) Q(t) .
$$

Since $J(T, x)=\frac{1}{2} x^{\tau} P(T) x+Q(T) x+K(T)=x^{\tau} \Psi_{T} x$, we have $P(T)=2 \Psi_{T}, Q(T)=0$, and $K(T)=0$. By Eq. (15), we obtain Eqs. (6) and (7). By Eq. (16), Eq. (8) holds. Therefore,

$$
J(t, x)=\frac{1}{2} x^{\tau} P(t) x+Q(t) x+K(t)
$$

is the optimal value of the uncertain linear system with input delay Eq. (2) and the quadratic criterion Eq. (3), and

$$
u_{t}^{*}=-R^{-1}(t) M_{i}^{\tau}(t) \sum_{i=1}^{p} B_{i}^{\tau}(t)(P(t) x+Q(t)) .
$$

Let us find the value of matrices $M_{i}(t)$ for this problem. Substituting the optimal control law Eq. (17) into the Eq. (2) gives

$$
\begin{aligned}
d X_{s}=\{ & -\sum_{i=1}^{p} B_{i}(s) R^{-1}\left(s-h_{i}\right) M_{i}^{\tau}\left(s-h_{i}\right) \sum_{i=1}^{p} B_{i}^{\tau}\left(s-h_{i}\right)\left(P\left(s-h_{i}\right) X_{s-h_{i}}\right. \\
& \left.\left.+Q\left(s-h_{i}\right)\right)+a_{0}(s)+a_{1}(s) X_{s}\right\} d s+b(s) d C_{s} .
\end{aligned}
$$

The multi-dimensional uncertain differential Eq. (18) is equivalent to the multidimensional uncertain integral equation

$$
\begin{aligned}
X_{t}=U(r, t) & \left\{-\int_{r}^{t} U(t, s)^{-1}\left\{\sum_{i=1}^{p} B_{i}(s) R\left(s-h_{i}\right)^{-1} M_{i}^{\tau}\left(s-h_{i}\right) \sum_{i=1}^{p} B_{i}^{\tau}\left(s-h_{i}\right)\right.\right. \\
& \left.\left.\left(P\left(s-h_{i}\right) X+Q\left(s-h_{i}\right)\right)+a_{0}(s)\right\} d s+\int_{r}^{t} U(t, s)^{-1} b(s) d C_{s}+X_{r}\right\},
\end{aligned}
$$

where $t, r \geq t_{0}$, and

$$
U(r, t)=\exp \left(-\int_{r}^{t} a_{1}(s) d s\right)
$$


and we know

$$
U\left(t-h_{i}, t\right)=\exp \left(-\int_{t-h_{i}}^{t} a_{1}(s) d s\right) .
$$

Since the integral terms in the right-hand side of Eq. (19) do not explicitly depend on $u_{t}$,

$$
\partial x_{t} / \partial u_{t}=U(r, t) \partial x_{r} / \partial u_{t} .
$$

It can be converted to

$$
\partial u_{t} / \partial x_{t}=\left(\partial u_{t} / \partial x_{r}\right) U(t, r) .
$$

Hence, the equality

$$
T u_{t}=K_{1} U(r, t) K_{2} x_{r}
$$

holds, where $T \in R^{n \times m}$ and $K_{1}, K_{2} \in R^{n \times n}$ can be selected the same for any $t, r \geq t_{0}$. Writing the last equality for $x_{t+h_{i}}, h_{i}>0$, we have

$$
T u_{t+h_{i}}=K_{1} U\left(r, t+h_{i}\right) K_{2} x_{r} .
$$

Thus,

$$
\left(\partial\left(T u_{t}\right) / \partial T u_{t+h_{i}}\right)=U(r, t)\left(U\left(r, t+h_{i}\right)\right)^{-1}=U\left(t+h_{i}, t\right),
$$

which leads to

$$
\left(\partial\left(T u_{t}\right) / \partial u_{t+h_{i}}\right)=U\left(t+h_{i}, t\right) T .
$$

Now setting $T=B_{i}(t)$ and using $t-h_{i}$ instead of $t$ yields

$$
B_{i}(t)\left(\partial u_{t-h_{i}} / \partial u_{t}\right)=B_{i}(t) M_{i}(t)=U\left(t, t-h_{i}\right) B_{i}(t)=\exp \left(-\int_{t-h_{i}}^{t} a_{1}(s) d s\right) B_{i}(t),
$$

for $t \geq t_{0}+h_{i}$.

So,

$$
M_{i}(t)=\exp \left(-\int_{t-h_{i}}^{t} a_{1}(s) d s\right) .
$$

The theorem is proved.

\section{Example}

We consider the following example of uncertain linear systems with multiple time-delays in the control input

$$
\left\{\begin{array}{l}
J\left(0, X_{0}\right)=\sup _{u \in U} E\left(\frac{1}{2} \int_{0}^{2}\left(u_{s}^{2}+X_{s}^{2}\right) d s+X_{T}^{2}\right), \\
\text { subject to } \\
d X_{t}=\left(X_{t}+u_{t-0.1}+u_{t}\right) d t+d C_{t}, t \in[0,2] \\
u_{t}=0, t \in[-0.1,0] \\
X_{0}=1 .
\end{array}\right.
$$

We have $a_{0}(t)=0, a_{1}(t)=1, B(t)=1, b_{0}(t)=0, b_{1}(t)=1, R(t)=1, L(t)=1$, and $\Psi(T)=1$. So, we get $M_{1}=\exp (-0.1)$ and $M_{2}(t)=1$. By Theorem 3.1, the function $Q(t)$ satisfies

$$
\left\{\begin{array}{l}
\frac{d Q(t)}{d t}=-(1+\exp (-0.1))^{2} P(t) Q(t)+Q(t) \\
Q(2)=0
\end{array}\right.
$$


Table 1 Numerical solutions

\begin{tabular}{llllllll}
\hline$t$ & 0 & 0.1 & 0.2 & 0.3 & 0.4 & 0.5 & \multicolumn{1}{c}{0.6} \\
\hline$x(t)$ & 1.000000 & 0.996292 & 1.008533 & 0.960251 & 0.968829 & 0.967966 & 0.967966 \\
$P(t)$ & 1.067544 & 1.067548 & 1.067554 & 1.067565 & 1.067586 & 1.067624 & 1.067692 \\
$u *(t)$ & -2.033493 & -1.922702 & -2.025984 & -2.050895 & -1.952756 & -1.970269 & -1.968630 \\
$t$ & 0.7 & 0.8 & 0.9 & 1.0 & 1.1 & 1.2 & 1.3 \\
$x(t)$ & 0.976390 & 0.964772 & 0.977926 & 0.944681 & 0.940976 & 0.944992 & \multicolumn{1}{l}{0.929304} \\
$P(t)$ & 1.067815 & 1.068038 & 1.068441 & 1.069075 & 1.070317 & 1.072567 & 10.7665 \\
$u *(t)$ & -1.985991 & -1.962773 & -1.990284 & -1.92376 & -1.918448 & -1.930682 & -1.905861 \\
$t$ & & & & & & & \\
$x(t)$ & 1.4 & 1.5 & 1.6 & 1.7 & 1.8 & 1.9 & 2.0 \\
$P(t)$ & 1.08408163 & 0.86307 & 0.873138 & 0.856087 & 0.784042 & 0.744824 & 0.6788663 \\
$u *(t)$ & -1.871245 & -1.097717 & 1.11969 & 1.164538 & 1.252615 & 1.439701 & 2.000000 \\
\hline
\end{tabular}

Thus, $Q(t)=0$ for $t \in[0,2]$, and then $K(t)=0$ for $t \in[0,2]$. So, we get that the optimal control $u_{t}^{*}$ is

$$
u_{t}^{*}=-(1+\exp (-0.1)) P(t) x,
$$

and the optimal value is $J\left(0, X_{0}\right)=\frac{1}{2} P(0) X_{0}^{2}$, and $P(t)$ satisfies

$$
\left\{\begin{array}{l}
\frac{d P(t)}{d t}=-(1+\exp (-0.1))^{2} P(t)^{2}+2 P(t)+2 \\
P(2)=2 .
\end{array}\right.
$$

Now, we consider the numerical solution of this model. Let $S=t_{0}, t_{1}, \cdots t_{200}$ be an average partition of [0,2](i.e., $0=t_{0}<t_{1}<\cdots<t_{200}=2$ ), and $\Delta t=0.01$. Thus,

$$
\Delta X_{t}=\left(X_{t}+u_{t-0.1}^{*}+u_{t}^{*}\right) \Delta t+\Delta C_{t} .
$$

Since $\Delta C_{t}$ is a normal uncertain variable with expected value 0 and variance $\Delta t^{2}$, the distribution function is $\Phi(x)=\left(1+\exp \left(-\frac{\pi x}{\sqrt{3} \Delta t}\right)\right)^{-1}, x \in R$. So, we maybe can get a

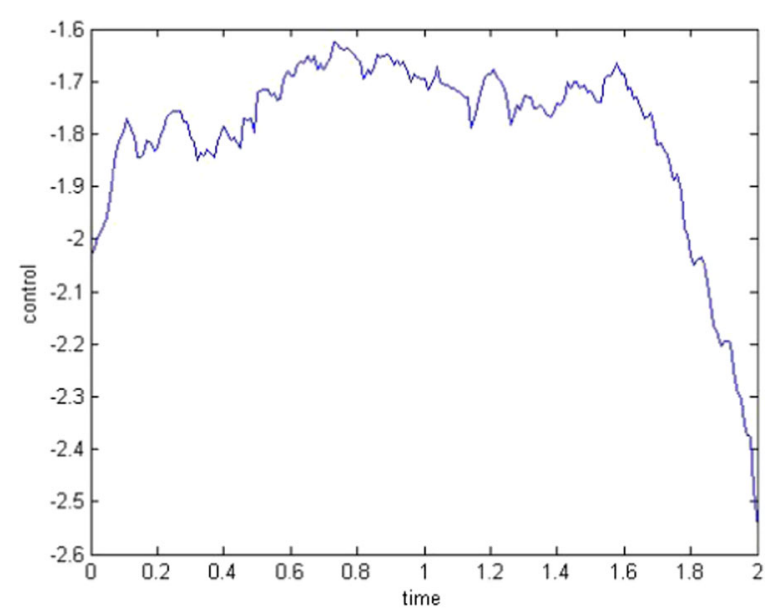

Fig. 1 Optimal control with time 


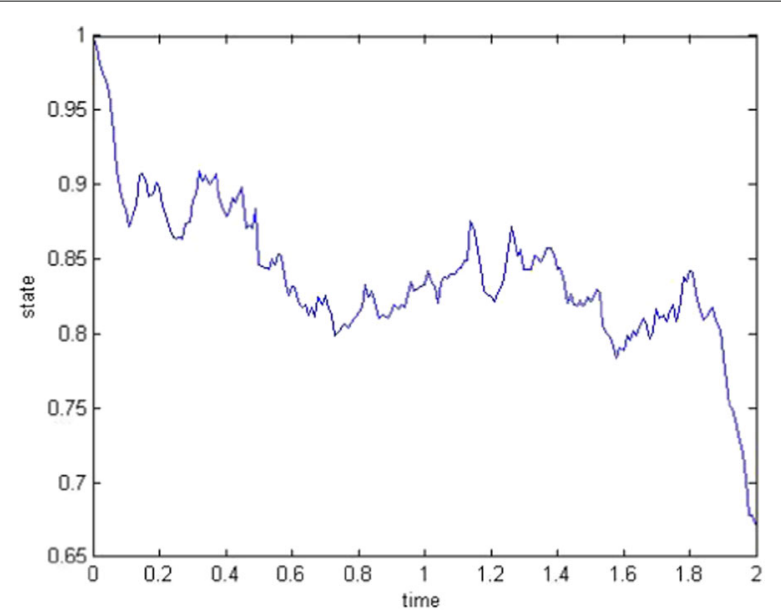

Fig. 2 Corresponding state with time

sample point $\tilde{c_{t}}$ of $\Delta C_{t}$ from $\tilde{c_{t}}=\Phi^{-1}(\operatorname{rand}(0,1))$ that $\tilde{c_{t}}=\frac{\sqrt{3} \Delta t}{-\pi} \ln \left(\frac{1}{\operatorname{rand}(0,1)}-1\right)$. Thus, $x_{t}$ and $u_{t}$ may be given by the following iterative equations:

$$
\begin{aligned}
& u_{t_{j}}=-(1+\exp (-0.1)) P\left(t_{j}\right) x_{t_{j}}, \\
& x_{t_{j+1}}=x_{t_{j}}+\left(x_{t}+u_{t_{j}-0.1}+u_{t_{j}}\right) \Delta t+\frac{\sqrt{3} \Delta t}{-\pi} \ln \left(\frac{1}{\operatorname{rand}(0,1)}-1\right),
\end{aligned}
$$

for $j=0,1,2, \cdots, 200$, and $u_{t_{j}-0.1}=0$, where $t_{j} \in[0,0.1]$. The numerical solution $P\left(t_{j}\right)$ of (23) is provided by

$$
P\left(t_{j-1}\right)=P\left(t_{j}\right)-\left(-(1+\exp (-0.1))^{2} P\left(t_{j}\right)^{2}+2 P\left(t_{j}\right)+2\right) \Delta t,
$$

for $j=200,199, \cdots, 2,1$ with $P\left(t_{200}\right)=2$.

Therefore, the optimal value of the example is $J\left(0, X_{0}\right)=1.067544$, and the optimal controls and corresponding states are obtained in Table 1 and Figs. 1 and 2.

\section{Conclusion}

In this paper, an optimal control problem for uncertain linear systems with multiple input delays was investigated. By using uncertain optimality equation and uncertain differential equation, then the optimal control of this problem was obtained. Finally, an example was used to illustrate the result of uncertain optimal control.

Acknowledgements

This work is supported by the National Natural Science Foundation of China (No. 61273009, No. 61673011).

\section{Authors' contributions}

YJ carried out the study in the paper and drafted the first version of the manuscript. YJ and YZ designed the framework together. $\mathrm{HY}$ and $\mathrm{YZ}$ revised the first version of the manuscript. All authors read and approved the final manuscript.

\section{Competing interests}

The authors declare that they have no competing interests.

\section{Author details}

${ }^{1}$ School of Science, Nanjing University of Science and Technology, 210094 Nanjing, China. ${ }^{2}$ School of Science, Nanjing Forestry University, 210037 Nanjing, China.

Received: 1 July 2016 Accepted: 19 September 2016

Published online: 06 October 2016 


\section{References}

1. Basin, M: Optimal control problems for time-delay systems, 380, 131-173. Springer, Berlin (2008)

2. Eller, D, Aggarwal, JK, Banks, H: Optimal control of linear time-delay systems. IEEE Trans. Autom. Control. 14(6), 678-687 (1970)

3. Bauer, H, Rieder, U: Stochastic control problems with delay. Math. Methods Oper. Res. 62(3), 411-427 (2005)

4. Larssen, B, Risebro, N: When are HJB-equation for control problems with stochastic delay equation finite dimensional? Stoch. Anal. Appl. 21, 643-671 (2003)

5. Yong, J, Zhou, X: Stochastic controls: Hamiltonian systems and HJB equations. Springer, New York (1999)

6. Liu, B: Uncertainty theory. 2nd ed. Springer-Verlag, Berlin (2007)

7. Liu, B: Fuzzy process, hybrid process and uncertain process. Journal of Uncertain Systems. 2(1), 3-16 (2008)

8. Liu, B: Some research problems in uncertainty theory. J Uncertain Syst. 3(1), 3-10 (2009)

9. Ji, X, Zhou, J: Multi-dimensional uncertain differential equation: existence and uniqueness of solution. Fuzzy Optim. Decis. Making. 14(4), 477-491 (2015)

10. Zhu, Y: Uncertain optimal control with application to a portfolio selection model. Cybern. Syst. Int. J. 41(7), 535-547 (2010)

11. $\mathrm{Xu}, \mathrm{X}, \mathrm{Zhu}, \mathrm{Y}$ : Uncertain bang-bang control for continues time model. Cybern. Syst. Int. J. 43(6), 515-527 (2012)

12. Chen, R, Zhu, Y: An optimal control model for uncertain systems with time-delay. J. Oper. Res. Soc. Jpn. 4(2013), 243-256

\section{Submit your manuscript to a SpringerOpen ${ }^{\circ}$ journal and benefit from:}

- Convenient online submission

- Rigorous peer review

- Immediate publication on acceptance

- Open access: articles freely available online

- High visibility within the field

Retaining the copyright to your article

Submit your next manuscript at $\gg$ springeropen.com 